\title{
68. Untersuchungen über Kohlehydrate von den Scrophulariaceen.
}

\author{
I. Mitteilung. Über das Vorkommen der Stachı̣nse \\ in der Wurzel von Scrophularia \\ kakudensis Franchet.
}

Von Susumu Murakami.

Iwata Institut für Pflanzenbiochemie in Tokyo.

(Comm. by K. Shibata, M.I.A., May 12, 1944.)

In einer früheren Mitteilung habe ich die Verbreitung der Stachyose, eines zinerst von A. Planta und E. Schulze ${ }^{1)}$ in den Japanknollen aufgefundenen Tetrasaccharids, in den Lippenblütlern beschrieben ${ }^{2}$. Derselbe Zucker (Lupeose) soll auch in den Samen einiger Leguminosen vorkommen ${ }^{3}$.

Nun gelang es mir, die Stachyose auch aus den Wurzeln von Scrophularia kakudensis Franchet und einigen anderen ScrophulariaArten $\mathrm{zu}$ isolieren.

Zur Darstellung der Stachyose wurde das zerkleinerte Pflanzenmaterial mit 40-proz. Alkohol ausgezogen. Nach dem Verjagen des Alkohols wurden die Auszüge mit Bleiessig enteiweisst und darauf mit Barytwasser und Alkohol versetzt. Die gewonnenen Niederschläge wurden durch Kohlensäurestrom zerlegt. Nach wiederholten Umfällungen aus wässriger Lösung mit Alkohol wurde die Stachyose in tafelförmigen Krystallen erhalten (Fig. 1).

Die so gereinigte Stachyose löst sich leicht in Wasser, dagegen schwer in Alkohol, schmeckt süss, reduziert Fehlingsche Lösung nicht. Sie schmilzt bei $170^{\circ}$ und zeigt eine spezifische Drehung $[\alpha]_{D}^{5}=+147.84^{\circ}$. Die Elementaranalyse entspricht der Bruttoformel $\mathrm{C}_{24} \mathrm{H}_{42} \mathrm{O}_{21}(=666)$ und das kryoskoplsch gemessene Molekulargewicht ist mit der Formel eines Tetrasaccharides gut vereinbar.

Das Acetylderivat wurde mit Pyridin und Essigsäureanhydrid dargestellt. Es schmilzt bei $97^{\circ}$, zeigt $[\alpha]_{D}^{6}=+118.39^{\circ}$ (Alkohol) und stimmt sowohl nach der Elementaranalyse als auch nach der Kryoskopie in Benzol mit der Formel $\mathrm{C}_{24} \mathrm{H}_{28} \mathrm{O}_{21}\left(\mathrm{CH}_{3} \mathrm{CO}\right)_{14}$.

Als konstituierende Zucker der Stachyose kommen nach qualitativer Untersuchung nur Fructose, Glucose und Galactose in Betracht. Hierbei wurde Fructose nach Seliwanoffscher Reaktion und Glucose und Galactose

1) A. W. Planta u. E. Schulze: Ber. Dtsch. chem. Ges., 23 (1890), $1692 ; 24$ (1891), 2705 ; Landwirtschaftl. Vers.-Stat., 40 (1892), 277 ; 41 (1893), 123. Übe. ¿̈.: Konstitutionsbestimmung der Stachyose als Galactosido-galactosido-glucosido-fructosid vgl. M. Onuki : Bull. Inst. phys.-chem. Res., Tokyo, 12 (1933), 35.

2) Acta Phytochim., 13 (1943), 161. Vgl. auch L. Piault: Journ. Pharm. Chim., (7) 1 (1910): 248.

3) C. Tanret: Compt. rend., 155 (1912), 1562; Bull. Soc. Chim. France, (4) 13 (1913) 176 ; H. Herissey u. R. Sibassié : Compt. rend., 178 (1924), 884; H. Colin u. R. Franquet: Compt. rend., 187 (1928), 309 ; E. Steiger: Ber. Dtsch. chem. Ges., 25 (1892), 2213; 43 (1910), 2230. 
durch Oxydation mit Salpetersäure als Zuckersäure bzw. Schleimsäure identifiziert.

Nach der Hydrolyse mit $n$-Schwefelsäure erniedrigte sich die spezifische Drehung einer Stachyoselösung von $+127.95^{\circ} \mathrm{zu}+37.80^{\circ}$. Der Reduktionswert dieses Hydrolysates wurde nach Bertrand und der Aldosewert nach Willstätter und Schudel ermittelt, wonach die Hydrolyse zur Stufe der Hexosen beinah vollständig war.



Fig. 1. Stachyose. $(\times 40$. $)$

Durch die bei $80^{\circ}$ ausgeführte, 5-stündige Hydrolyse mit 20-proz. Essigsäure wurde die spezifische Drehung der Lösung von $+130^{\circ} \mathrm{zu}$ $+94^{\circ}$ herabgedrückt. Das Hydrolysat wurde nun nach Neutralisation zur Syrupdicke eingeengt und mit Alkohol versetzt. Der in Alkohol lösliche Teil zeigte eine Linksdrehung und gab mit Resorcin und Salzsäure eine starke Seliwanoffsche Reaktion. Der mit Alkohol gefällte Teil wurde nach wiederholter Umfällung in Form eines schneeweissen Pulvers gewonnen. Es reduzierte Fehlingsche Lösung und gab mit Phenylhydrazinchlorhydrat und Natriumacetat das Manninotriosazon vom Schmelzpunkt $122^{\circ}$.

Nach den oben angeführten Ergebnissen kann der Zucker aus Scrophularia kakundensis als die Stachyose genügend gut charakterisiert werden.

\section{Beschreibung der Versuche.}

Die im Oktober gesammelte, frische Wurzel von Scrophularia kakudensis Franchet wurde zerhackt und zweimal mit 40-proz. Alkohol unter Rückfluss auf dem Wasserbad erhitzt und dann ausgepresst. Die vereinigten Auszüge wurden nach Verjagung des Alkohols wie üblich mit Bleizucker und basischem Bleiacetat gefällt. Das Filtrat wurde nach der Entfernung von gelösten Bleisalzen mittels Schwefelwasserstoffs mit Ammoniak neutralisiert und auf dem Wasserbade eingeengt. Die Lösung wurde dann abwechselnd mit Bariumhydroxyd und Alkohol 
versetzt. Der entstandene weisse Niederschlag wurde gründlich mit bariumhydroxydhaltigem Alkohol nachgewaschen, in einer Reibschale mit Wasser gut zerrieben und durch einen lebhaften Kohlensäurestrom zersetzt. Aus dem Filtrat vom Bariumcarbonat wurde die Stachyose. durch Zufügen von Alkohol zur Ausscheidung gebracht. Nach wiederholter Umfällung aus wässriger Lösung mit Alkohol wurden die tafelförmigen Stachyosekrystalle erhalten. Ausbeute 3.7 Proz. des Ausgangsmaterials.

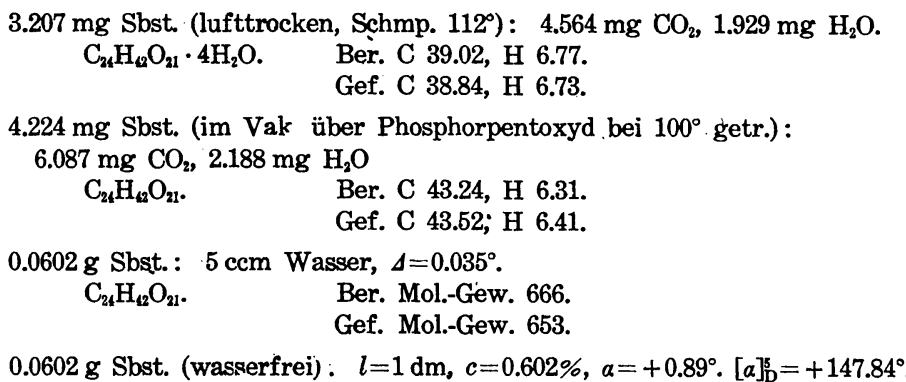

Die Substanz ist in Wasser leicht, dagegen in Alkohol schwer löslich, schmeckt süss, reduziert Fehlingsche Lösung nicht und ist dialysierbar durch Fischblase. Mit Barytwasser und Alkohol wird sie nur aus konzentrierter Lösung und mit Strontiumhydroxydlösung in der Hitze gefällt.

Prüfung auf Galactose : $0.5 \mathrm{~g}$ Substanz wurden in $30 \mathrm{ccm}$ Salpetersäure $(d 1.15)$ gelöst und auf dem Wasserbade bei $80^{\circ}$ oxydiert. Die bis auf ein Drittel des Volumens eingeengte Lösung wurde abgekühlt, die ausgeschiedene Schleimsäure mit Wasser, Alkohol und Äther gewaschen. Die Krystalle wurden in wenig verdünnter Natronlauge gelöst und durch Ansäuerung mit Salzsäure wieder zur Krystallisation gebracht. Die Ausbeute an Schleimsäure betrug $0.135 \mathrm{~g}$ und entspricht $0.207 \mathrm{~g}$ Galactose (41\% des Ausgangsmaterials).

Prüfung auf Glucose: Die eingedampfte syrupöse Mutterlauge von Schleimsäure wurde mit Kaliumcarbonat neutralisiert unu nach Zusatz von einigen Tröpfchen Essigsäure zum Syrup eingedunstet. Nach einer Nacht Stehen schied sich aus diesem das Krystallisat von zuckersaurem Kalium.

Prüfung auf Fructose: Die wässrige Lösung des Zuckers wurde mit gleichem Volumen 25-proz. Salzsäure und einigen Kryställchen von Resorcin auf dem Wasserbade erhitzt. Nach einigen Minuten erschien eine rote Färbung und dann ein Niederschlag.

Säurehydrolyse der Stachyose: (A) $0.2751 \mathrm{~g}$ Substanz wutden in $20 \mathrm{ccm} n$-Schwefelsäure gelöst und im Wasserbade auf $90^{\circ}$ am Rückflusskühler erhitzt. Nach 1, 2, 3 Stunden zeigte sich die Drehung als $+43.62^{\circ},+37.80^{\circ},+37.80^{\circ}$.

Das Hydrolysat wurde mit Bariumhydroxyd neutralisiert und eingeengt. Der Syrup wurde mit $0.5 \mathrm{~g}$ Methylphenylhydrazin, $10 \mathrm{ccm}$ Alkohol und $1 \mathrm{ccm}$ 50-proz. wässriger Essigsäure versetzt und bei Zimmertemperatur stehen gelassen, wobei sich Methylphenylhydrazon 
Reduktionswert (Probe je $2 \mathrm{ccm}, 27.51 \mathrm{mg}$ Sta nyose enthaltend).

\begin{tabular}{c|c|c|c|c|c}
\hline \multicolumn{3}{c|}{ Nach Bertrand } & \multicolumn{3}{c}{ Nach Willstätter und Schudel } \\
\hline $\begin{array}{c}n / 10 \mathrm{KMnO} \\
\mathrm{ccm}\end{array}$ & $\begin{array}{c}\text { Hexose } \\
\mathrm{mg}\end{array}$ & $\begin{array}{c}\text { Spaltungs- } \\
\text { grad \% }\end{array}$ & $\begin{array}{c}n / 10 . \mathrm{Na}_{2} \mathrm{~S}_{2} \mathrm{O}_{3} \\
\mathrm{ccm}\end{array}$ & $\begin{array}{c}\text { Aldose } \\
\text { mg }\end{array}$ & $\begin{array}{c}\text { Spaltungs- } \\
\text { grad \% }\end{array}$ \\
\hline 9.0 & 28.9 & 100 & 2.4 & 21.6 & 78 \\
\hline
\end{tabular}

der Galactose abschied. Aus dem Filtrat wurde mit Phenylhydrazinchlorhydrat und Natriumacetat Glucosazon vom Schmp. $203^{\circ}$ erhalten.

(B) $0.2 \mathrm{~g}$ Substanz wurden in $20 \mathrm{ccm} 20$-proz. Essigsäure, gelöst und im Wasserbade auf $80^{\circ}$ am Rückflusskühler erhitzt. Nach 5 Stunden zeigte die Lösung eine Drehung $+94^{\circ}$. Die gelöste Essigsäure wurde dann durch Schütteln mit Äther möglichst gut beseltigt und die Lösung wurde, nachdem sie zur Syrupdicke eingedampft war, mit Alkohol versetzt. Der in Alkohol lösliche Teil erwies sich als linksdrehend und gab mit Resorzin und Salzsäure eine stark positive Seliwanoffsche Reaktion. Der mit Alkohol gefällte Teil wurde nach wiederholter Umfällung in Form eines schneeweissen Pulvers erhalten, welches bei $146^{\circ}$ schmolz, Fehlingsche Lösung reduzierte und mit Phenylhydrazinchlorhydrat und Natriumacetat das Phenylosazon von Manninotriose vom Schmp. 122 lieferte.

\section{Acetylderivat.}

$0.2 \mathrm{~g}$ Substanz wurden in $5 \mathrm{ccm}$ Pyridin bei $70^{\circ}$ der Quellung über. lassen und dann mit $5 \mathrm{ccm}$ Essigsäureanhydrid versetzt. Das Gemisch wurde nach einer Woche Stehen ins Eiswasser hineingegossen. Das abfiltrierte Produkt wurde mehrmals aus Eisessig und warmen Methylalkohol umgefällt und bildete ein weisses, sehr feines Pulver vom Schmp. $97^{\circ}$.

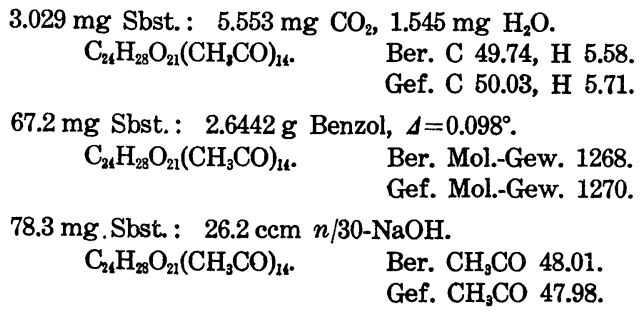

$119.1 \mathrm{mg}$ Sbst. : $l=1 \mathrm{dm}, c=0.794 \%, a=+0.94^{\circ} .[a]_{\mathrm{D}}^{6}=+118.39^{\circ}$ (Alkohol).

Zum Schluss möchte ich meinem verehrten Lehrer, Herrn Ehrenprof. Dr. K. Shibata, für seine gütige Leitung und stete Anregung meinen herzlichsten Dank aussprechen. 